\title{
Eccentric compression properties of laminated bamboo lumber columns with different slenderness
}

\section{ratios}

\author{
Hai-tao $\mathrm{LI}^{\mathrm{a}, \mathrm{b}^{* 1}}$, Rong Liu ${ }^{\mathrm{a}}$, Rodolfo Lorenzo ${ }^{\mathrm{c}}$, Gang Wu ${ }^{\mathrm{b}}$, Li-bin WANG ${ }^{\mathrm{a}}$ \\ ( ${ }^{a}$ Collage of Civil Engineering, Nanjing Forestry University, Nanjing, 210037, China) \\ ( ${ }^{\mathrm{b} S o u t h e a s t}$ University, Key laboratory of concrete and pre-stressed concrete structure of \\ Ministry of Education (College of Civil Engineering), Nanjing, 210096, China) \\ ( ${ }^{c}$ University College London, London WCIE 6BT, UK)
}

\begin{abstract}
Slenderness ratio is one of the main influencing factors on the mechanical properties of LBL column under eccentric compression. This paper presents the research results of 20 column tests. Two main failure models can be classified based on the test results for the LBL columns. The increasing speeds for the strain value are similar for all specimens. Regardless of the length, the strain across the cross-section of the LBL column for each specimen is basically linear throughout the loading process, following standard normal section bending theory. The relationships between the ultimate mechanical parameters and slenderness ratios were proposed, including an equation for calculating slenderness ratio influencing coefficient $\varphi_{\lambda}$ which gives a good agreement with the test results. All the equations presented in this paper can be used as reference for further work to establish a generally applicable formula for code adoption.
\end{abstract}

Key words: Building, structures \& design; Columns; Timber structures

\section{Notation}

A the area of the cross section

$H$ the height from the bottom to the calculation point of the column

$i \quad$ the gyration radius of the cross section

$I \quad$ the inertia moment of the cross section

$l_{0} \quad$ the calculation length of the LBL column

$L \quad$ the length of the column

$M \quad$ the moment.

${ }^{1}$ Corresponding author: Hai-tao LI, Associate Professor, Post-Doctoral Research Fellow; E-mail: lhaitao1982@126.com 
$M_{\mathrm{ul}} \quad$ the ultimate moment.

$N_{\text {ul }}^{\mathrm{c}} \quad$ the test ultimate load

$N_{\mathrm{ul}}^{\mathrm{c}} \quad$ the calculated ultimate load

$N_{0} \quad$ the ultimate bearing capacity

$s \quad$ the axial displacement

$s_{\mathrm{ul}} \quad$ the ultimate axial displacement

$w \quad$ the deflection of the LBL column

$w_{\mathrm{m}} \quad$ the middle deflection value of the column

$w_{\mathrm{ul}} \quad$ the ultimate middle deflection for the peak load point

$\varepsilon_{\text {uasA }}$ the longitudinal strain for face A

$\varepsilon_{\text {uasC }}$ the longitudinal strain for face $\mathrm{C}$

$\varepsilon_{\text {ulsA }}$ the lateral strain for face A

$\varepsilon_{\text {ulsC }}$ the lateral strain for face C

$\varepsilon_{\text {uasD }}$ the ultimate axial strain for face D

$\varepsilon_{\text {ulsD }}$ the ultimate lateral strain for face D

$\lambda \quad$ the slenderness ratio

$\rho \quad$ the curvature radius

$\phi \quad$ the curvature for the middle cross-section

$\varphi_{\lambda} \quad$ the slenderness ratio influencing coefficient

$\mu \quad$ the mean value of $N_{\mathrm{ul}}^{\mathrm{t}} / N_{\mathrm{ul}}^{\mathrm{c}}$

\section{Introduction}

Bamboo as a structural material is attracting increased scientists' attention due to its favorable physical, mechanical and environmental characteristics. However, bamboo poles have important limitations: e.g. susceptibility to splitting, available diameters, ease (or difficulty) of connection and limited fire resistance. Engineered bamboo products such as those shown in Fig. 1 have been previously studied as an alternative to address these limitations (Chen et al. 2014; Huang et al. 2016; Sulaiman et al. 2006; Zhong et al. 2016). Laminated bamboo lumber (LBL, Fig.1a) is one particular kind of engineered bamboo product which has been the focus of previous research (Nugroho et al. 2001; Mahdavi et al. 
2011). As described by Li et al. (2015b, 2018), the manufacturing of LBL involves the processing of bamboo culm into thin flat elements which are subsequently laminated together with adhesive to form certifiable structural members. Both the cross section and length can be accurately controlled in a factory environment (Li et al. 2016a, 2016b, 2016c).

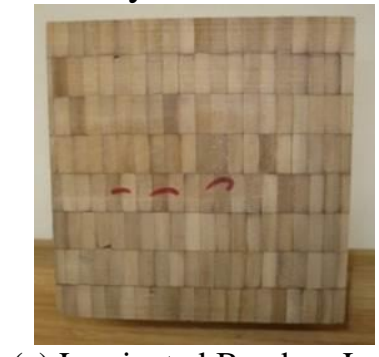

(a) Laminated Bamboo Lumber

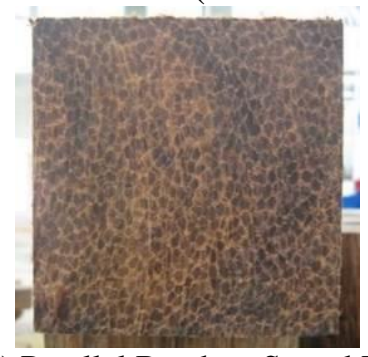

(b) Parallel Bamboo Strand Lumber

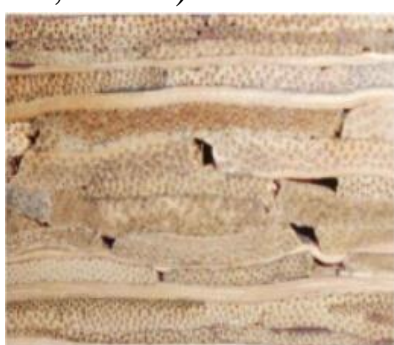

(c) Glubam

Considering many influencing factors, basic mechanical properties have been investigated (Sharma et al. 2015; Verma et al. 2012) including the tensile, compressive, shear and bending properties of small specimens (Correal et al. 2010, 2014; Yeh and Lin, 2012; Lee et al. 2012; Lee et al. 1998). Su et al. (2015b) have conducted experimental research on parallel bamboo strand lumber (PBSL, Fig.1b) column under axial compression. Li et al. (2015a) and Wei et al. (2016) investigated how the eccentricity values influence the eccentric compression performance of PBSL column respectively. Xiao and Shan (2013) have studied glubam (Fig.1c) columns under axial compression. These previous studies have focused on the mechanical properties of PBSL and Glubam but studies on LBL column as structural elements are scarce. The study of Li et al. (2013) and Su et al. (2015a) showed that short LBL columns (with the cross section of $100 \mathrm{~mm}$ x $100 \mathrm{~mm}$ ) in compression display a significant amount of plastic behaviour before crushing, and also showed that the stress-strain relationship in compression could be represented using a tri-linear model with an elastic portion, and elasto-plastic portion and a purely plastic portion. Luna et al. (2013) and Li et al. (2015b) investigated the mechanical performance of LBL columns under axial compression considering the slenderness ratio. Considering the eccentricities, production technology and eccentric directions, Li et al. (2016d, 2016e, and 2016f) examined and compared the mechanical properties for LBL columns under different eccentric compression directions. As discussed above, there is a limited number of papers addressing the performance of LBL columns under eccentric compression, particularly considering the slenderness ratios.

To the authors' knowledge, few studies on LBL columns under eccentric compression conditions have been conducted even though almost all columns used in the building industry experience eccentric compression. As the shear strength parallel to the grain is weak, it is complicated to carry out the eccentric compression test. However, it is important to evaluate the eccentric compression performance of columns. This study aims to examine how the slenderness ratio influence the behaviour of LBL columns under eccentric compression based on 20 test specimens.

In order to understand the eccentric compression behaviour of LBL columns with different slenderness ratios, this study examines in detail their behaviour comparing the effect of different slenderness ratios on their mechanical properties.

\section{Materials and test methods}


Moso bamboo (Phyllostachys pubescens, from Fujian province) harvested at the age of 3-4 years has been selected for this study. The cross section of the individual bamboo strips elements for LBL is rectangular (Fig. 2) with dimensions of $8 \mathrm{~mm} \times 21 \mathrm{~mm}$. The length of all strips is the same as that of the column specimens which means that there are no mechanical connections along the longitudinal direction of the strips. Laminated bamboo lumbers were produced following the same procedure as described by Li et al (2013). The material final moisture content and density were $6.52 \%$ and $640.3 \mathrm{~kg} / \mathrm{m}^{3}$ respectively. According to the compression tests, the compression strength for the laminated bamboo is $80.4 \mathrm{MPa}$, with a modulus of elasticity of $9694 \mathrm{MPa}$ and Poisson's ratio of 0.33 .

Five groups of specimens were produced with the same cross-section of $80 \mathrm{~mm} \times 80 \mathrm{~mm}$ and eccentricity value of $37 \mathrm{~mm}$ but differing lengths of 850, 1100, 1300, 1500 and $1700 \mathrm{~mm}$. Each group consisted of four identical specimens for a total of 20 samples. The nomenclature followed to identify each specimen groups follows the format 'JZ+ length'. As can be seen from Fig. 2, bamboo has three main directions which are longitudinal, radial, and tangential. Eccentricity in the radial direction has been considered for all specimens in this study (Fig. $2 b)$.

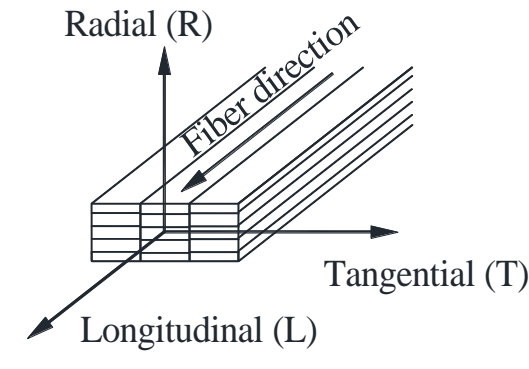

(a) Three main directions

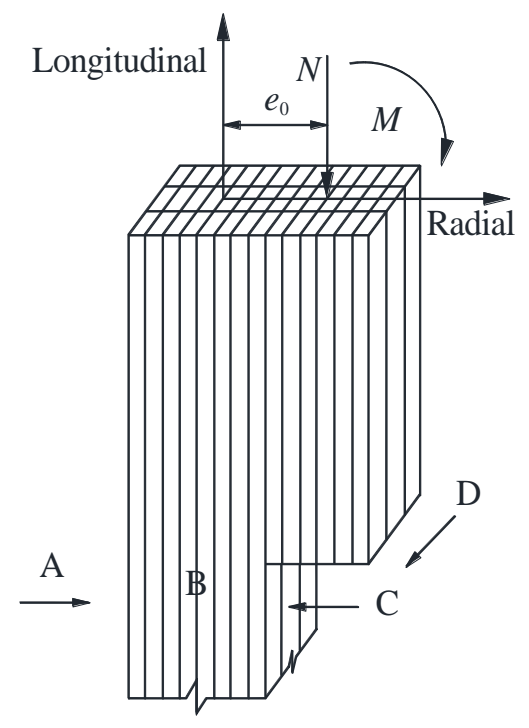

(b) Eccentric direction

Fig. 2. Specimen radial eccentric 


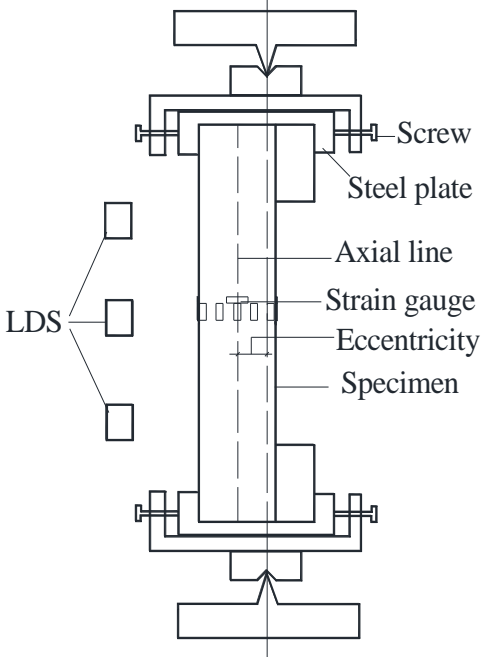

(a) Test configuration

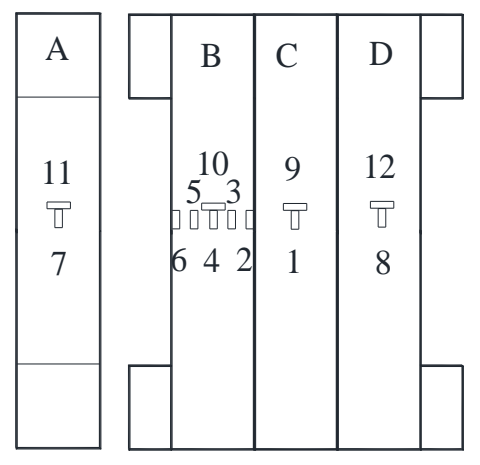

(b) Strain gauges arrangement (c) Test set-up

Fig. 3. Eccentric compressive test for columns

The displacements of the specimens along its axial direction at the quarter points and mid-span were measured by three Laser Displacement Sensors (LDS type: Keyence IL-300) respectively. Two strain gauges were installed centred on each of the specimens' sides with the exception of one side (Face D) where six strain gauges were installed. The strain gauges arrangement, including their identification number, is shown in Fig. 3. The compressive load was applied along the eccentricity line which is parallel to the axial line. The test was performed using a microcomputer-controlled electro-hydraulic servo universal testing machine with a capacity of $1000 \mathrm{kN}$ and a TDS Data Acquisition System.

The total loading duration was between 8-12 minutes. The load was applied initially through load control in the elastic stage with a speed of $800 \mathrm{~N} / \mathrm{s}$, and then was changed to displacement control before the proportional limit with a speed of $3 \mathrm{~mm} / \mathrm{min}$. The test continued at a constant displacement rate until the load reduced by $15 \%$ of the ultimate load, the middle deflection value was approximately $L / 15$ ( $L$ is the total length of the column) after the peak load point or the specimen had sustained significant damage, at which time testing was halted.

\section{Test results and analysis}

\subsection{Failure modes and mechanism analysis}

Two main failure models can be classified based on the test results. The first one is named failure mode I (Fig. 4(a)) in which the specimen splits firstly around the central position in face $\mathrm{C}$ (tensile side) and then the cracks appear, layer by layer, mainly from the tensile side towards the inner part of the specimen with the increase of loading. Except for face A, cracks can be clearly seen on the other three side surfaces. Most of the test specimens failed following the first mode. No obvious cracks appeared on the surface of the specimens for failure mode II (Fig. 4(b)). The test was stopped when the mid-length deflection value was approximately $75 \mathrm{~mm}$ after the peak load point but no clear failure phenomenon was visible on the surface. JZ1300-3 and all four specimens with length of $1700 \mathrm{~mm}$ belong to mode II. 
All specimens, regardless of their length, behaved elastically during the initial loading stage. The plastic deformation and the decrease in stiffness appeared when the loading value was about $80 \%$ of the ultimate load. Then the stiffness of the column decreased significantly as the loading increased. As for the specimens failed in mode I, cracks (accompanied by a slight noise) appeared on the tensile surface $\mathrm{C}$ as the deflection became obvious. However, no obvious cracks appeared for the specimens that failed in mode II but the ultimate lateral displacement was large. Finally, the load reduced by $15 \%$ of the ultimate load (mode I) or the middle deflection value was about $L / 15$ after the peak load point (mode II). The load-carrying capacity of the columns drops at this time and can be considered to have failed. Bending failure always occurred for all the column specimens under radial eccentric compression no matter how long the specimen was.

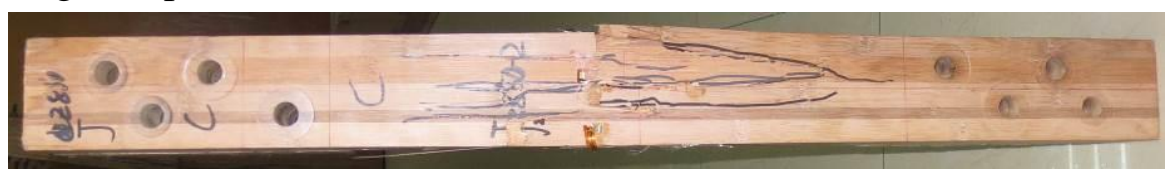

Face C

Face B

(a) Failure mode I (JZ850-2)

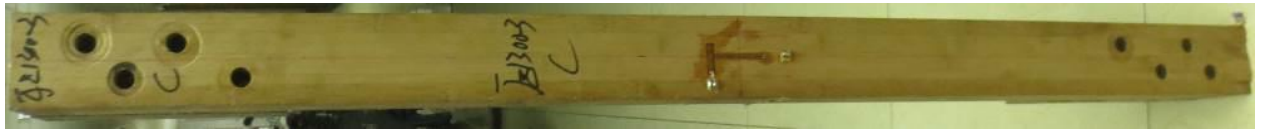

Face C

Face B

(b) Failure mode II (JZ1300--3)

Fig. 4. Typical failure modes for radial direction

Natural bamboo node is the main reason for the specimens' failure in mode I. Bamboo nodes are the weak point of the strips and the specimens crack firstly in the bamboo node area. All specimens show a large deflection at the end of the test.

As can be seen from Table 1, the average deflection at ultimate load is far larger than the maximum allowable design value $L / 250$ (where $L$ is the length of the column). Two critical design criteria (deflection and strength) are always used for structural members. Even though different failure modes happened, the column could still meet both design criteria.

\section{Table 1}


Test results

\begin{tabular}{|c|c|c|c|c|c|c|c|c|c|}
\hline Specimen & $N_{\mathrm{ul}}^{\mathrm{t}} / \mathrm{kN}$ & $w_{\mathrm{ul}} / \mathrm{mm}$ & $\varepsilon_{\text {uasC }} / \mu \varepsilon$ & $\varepsilon_{\text {uasA }} / \mu \varepsilon$ & $\varepsilon_{\mathrm{ulsC}} / \mu \varepsilon$ & $\varepsilon_{\mathrm{ulsA}} / \mu \varepsilon$ & $S_{\mathrm{ul}} / \mathrm{mm}$ & $M_{\mathrm{ul}} / \mathrm{kN} . \mathrm{m}$ & $N_{\mathrm{ul}}^{\mathrm{c}} / \mathrm{kN}$ \\
\hline JZ850-1 & 118.3 & 41.8 & 12402 & -12065 & -3323 & 6420 & 22.6 & 9.3 & 110.7 \\
\hline JZ850-2 & 111.4 & 42.5 & 12224 & -8755 & -2938 & 8720 & 19.5 & 8.9 & 110.7 \\
\hline JZ850-3 & 108.7 & 35.1 & 11062 & -10279 & -3467 & 6321 & 14.7 & 7.8 & 110.7 \\
\hline JZ850-4 & 105.9 & 36.7 & 11171 & -17956 & -3445 & 7444 & 15.8 & 7.8 & 110.7 \\
\hline JZ1100-1 & 85.2 & 48.4 & 10046 & -13881 & -2462 & 5431 & 18.1 & 7.3 & 84.3 \\
\hline JZ1100-2 & 77.0 & 60.9 & 12879 & -19841 & -3465 & 7062 & 23.6 & 7.5 & 84.3 \\
\hline JZ1100-3 & 84.7 & 52.8 & 10477 & -9668 & -1627 & 5294 & 18.9 & 7.6 & 84.3 \\
\hline JZ1100-4 & 84.2 & 56.8 & 11775 & -12364 & -3269 & 7371 & 22.2 & 7.9 & 84.3 \\
\hline JZ1300-1 & 70.1 & 68.0 & 11043 & -12075 & -3038 & 2582 & 21.9 & 7.4 & 70.8 \\
\hline JZ1300-2 & 68.0 & 70.1 & 10054 & -15542 & -2845 & 6077 & 19.9 & 7.3 & 70.8 \\
\hline JZ1300-3 & 71.9 & 73.4 & 11240 & -11066 & -2862 & 5044 & 25.9 & 7.9 & 70.8 \\
\hline JZ1300-4 & 73.0 & 68.1 & 10558 & -13292 & -2743 & 6555 & 23.8 & 7.7 & 70.8 \\
\hline JZ1500-1 & 59.7 & 83.6 & 10202 & -15288 & -2792 & 5381 & 25.8 & 7.2 & 61.0 \\
\hline JZ1500-2 & 63.5 & 74.4 & 9072 & -12338 & -2310 & 4473 & 23.8 & 7.1 & 61.0 \\
\hline JZ1500-3 & 65.0 & 90.7 & 11087 & -16039 & -3376 & 6197 & 28.3 & 8.3 & 61.0 \\
\hline JZ1500-4 & 64.0 & 79.9 & 9999 & -13649 & -2822 & 5262 & 23.9 & 7.5 & 61.0 \\
\hline JZ1700-1 & 54.2 & 99.4 & 10390 & -14999 & -2942 & 6090 & 30.2 & 7.4 & 53.6 \\
\hline JZ1700-2 & 55.4 & 97.6 & 8745 & -12818 & -2689 & 4951 & 27.4 & 7.5 & 53.6 \\
\hline JZ1700-3 & 50.4 & 98.1 & 9672 & -8427 & -2907 & 5424 & 26.2 & 6.8 & 53.6 \\
\hline JZ1700-4 & 50.8 & 77.9 & 9478 & -9741 & -2664 & 5882 & 30.1 & 5.8 & 53.6 \\
\hline
\end{tabular}

Note: $N_{\mathrm{ul}}^{\mathrm{t}}$ is the test ultimate load and $N_{\mathrm{ul}}^{\mathrm{c}}$ is the calculated ultimate load. $w_{\mathrm{ul}}$ is the ultimate middle deflection for the peak load point. $\varepsilon_{\text {uasA }}$ and $\varepsilon_{\text {uas } \mathrm{C}}$ are the longitudinal strain for face A and C respectively. $\varepsilon_{\text {ulsA }}$ and $\varepsilon_{\text {ulsC }}$ are the lateral strain for face A and C respectively. $S_{\mathrm{ul}}$ is the ultimate axial displacement. $M_{\mathrm{ul}}$ is the ultimate moment.

\subsection{Load strain curves comparison}

Fig. 5 and Fig. 6 show, respectively, the change in longitudinal and lateral strains at the centre of the specimens' side surfaces with the increase of loading. All strains displayed an initial elastic phase. For all specimens, face A experiences compression stresses along the longitudinal axial direction and tensile stress along the lateral direction at the middle cross section irrespective of how long the columns are. Meanwhile, tensile stresses develop at face $\mathrm{C}$ along the longitudinal axial direction and compression stresses along the lateral direction at the middle cross section also irrespective of the column length. In most cases, both the ultimate values for longitudinal strain and lateral strain for face A are bigger than those for face $\mathrm{C}$ independently of the column length. The reason is that fracture and failure due to the presence of defects happened firstly on the tensile side face $\mathrm{C}$ of the specimen. However, no clear failure happened to the compression side and face A could still undertake loading. Two kinds of strain values for face $\mathrm{B}$ and $\mathrm{D}$ are always consistent during the whole loading process no matter how long the columns are. As the columns become longer and longer, the increasing speeds for the strain value are similar for all specimens with the same eccentricity. 


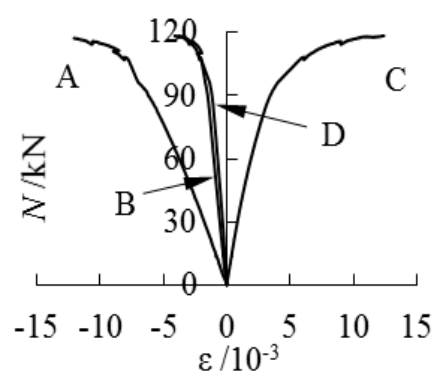

(a) JZ850-1

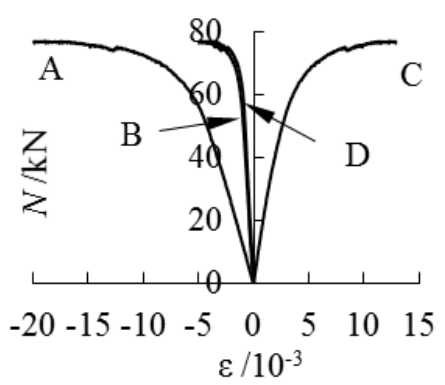

(b) JZ1100-2

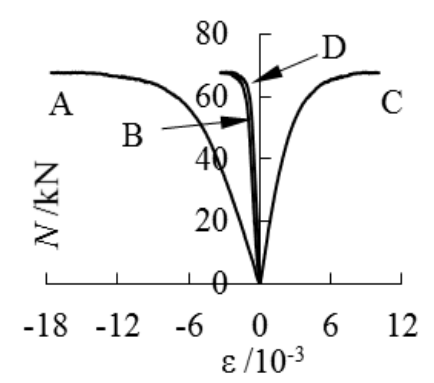

(c) JZ1300-2

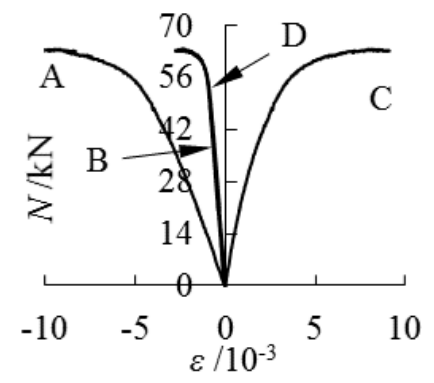

(d) JZ1500-2

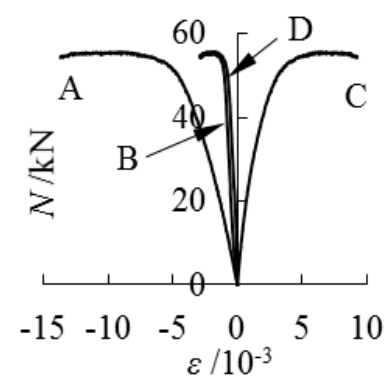

(e) JZ1700-2

Fig. 5. Load-longitudinal strain curves

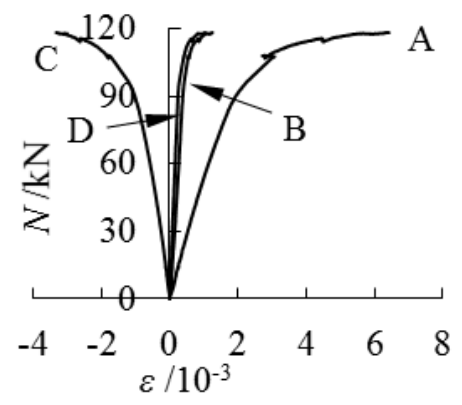

(a) JZ850-1

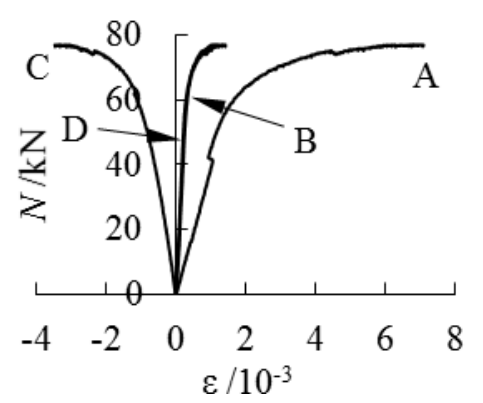

(b) JZ1100-2

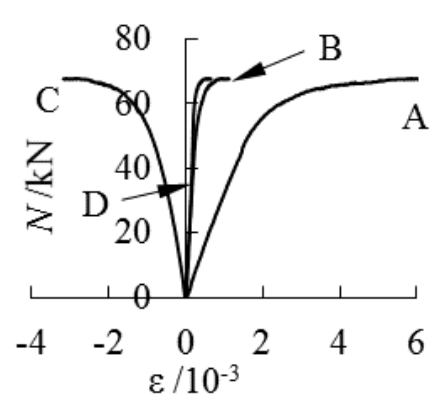

(c) JZ1300-2

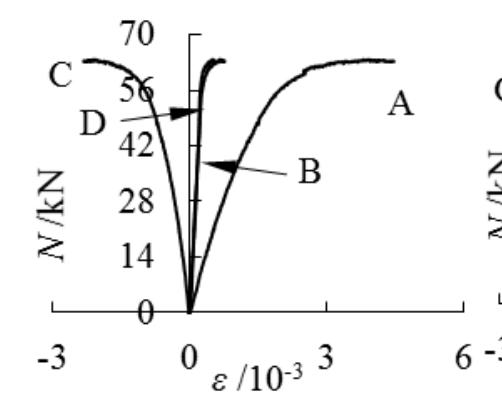

(d) JZ1500-2

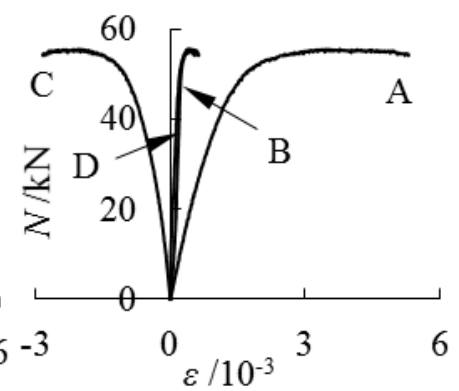

(e) JZ1700-2

Fig. 6. Load-lateral strain curves

\subsection{Strain distribution comparison}

Fig. 7 shows the typical evolution of the strain profile through the loading process for the mid-length cross-section of the specimens. It can be clearly seen that, no matter how long the columns are, the strain across the cross-section of the LBL column for each specimen is basically linear throughout the loading process, following standard normal section bending theory. The strain profile line is curved in the two ends as the compression side of the LBL materials becomes plastic. Even though all specimens have the same eccentricity, the neutral axis positions moves upwards slightly for increasing column lengths. This is thought to be 
due to the fact that the initial imperfection has greater influence on longer columns.

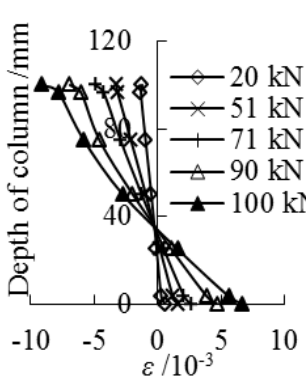

(a) JZ850-3

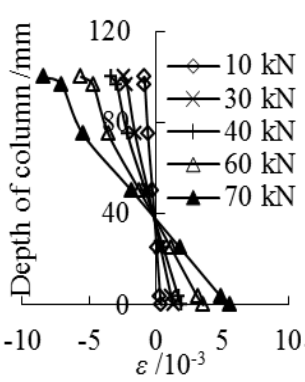

(b) JZ1100-2

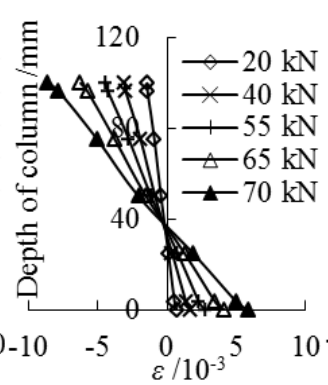

(c) JZ1300-4

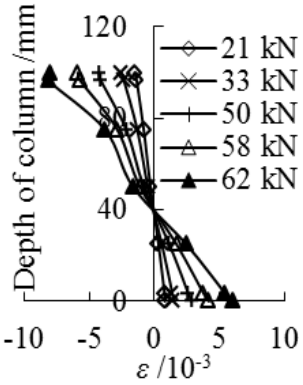

(d) JZ1500-2

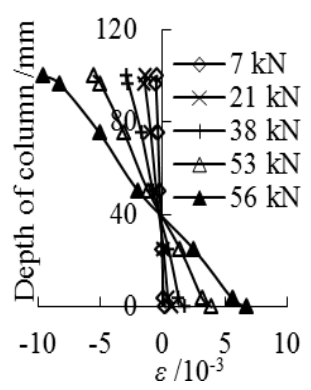

(e) JZ1700-2

Fig. 7. Typical strain profile at mid-length in the radial eccentric direction

\subsection{Displacement comparison}

Fig. 8 plots the typical load-lateral deflection curves under different load values. The trends were similar for columns irrespective of how long the columns are. Fitted sine half-wave curves were drawn using dotted lines in Fig. 8. It can be seen clearly that the measured deflections were close to the sine line no matter what the slenderness ratio was. The equation of the deflection curve can be expressed as (Eq. 1),

$$
w=w_{\mathrm{m}} \sin \frac{\pi H}{L}
$$

where $w$ is the deflection of the LBL column, $w_{\mathrm{m}}$ is the middle deflection value of the column, $H$ is the height from the bottom to the calculation point of the column, and $L$ is the total length of the column.

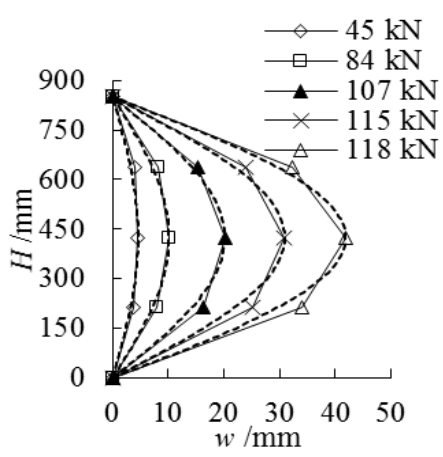

(a) JZ850-1

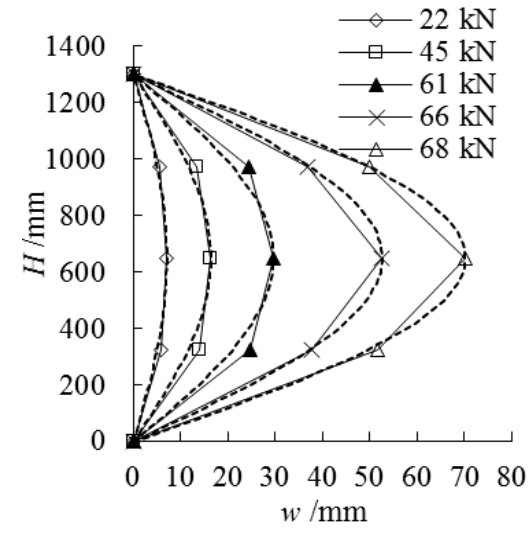

(b) JZ1300-2

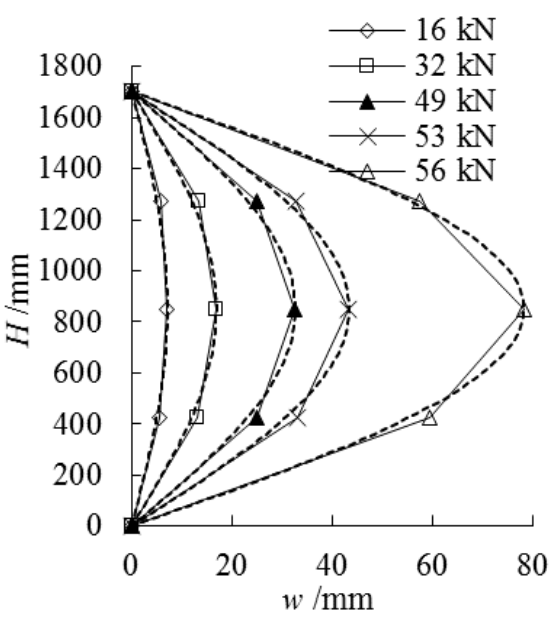

(c) JZ1700-2

Fig. 8. Load lateral deformation curves comparison
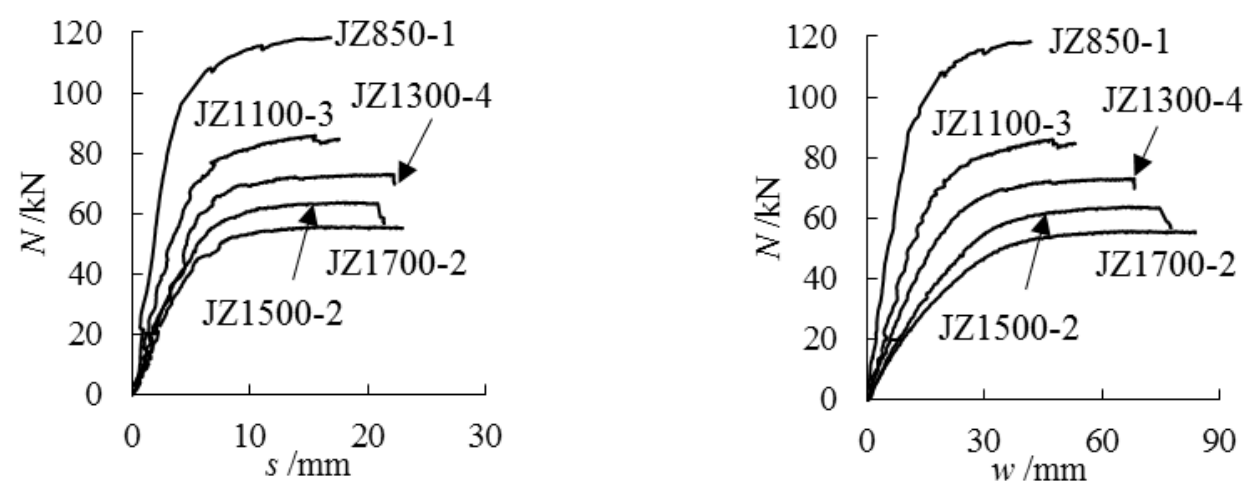

(a) Load - longitudinal displacement curves
(b) Load - middle deflection curves

Fig. 9. Typical load displacement curves comparison

The typical load against the displacement curves comparison can be seen from Fig. 9, where $s$ is the longitudinal displacement and $w$ is the lateral middle deflection. As shown in the figure, the specimens are under elastic compression in the initial stage, followed by no-linear behaviour irrespective of the column length. After the proportional limit in the curves, both the longitudinal displacement and the lateral deflection increase very quickly due to the secondary effect. The rate of increase in the displacement is more pronounced for longer columns before achieving the proportional limit. The longer the specimen, the bigger the displacement. In addition, the values for lateral middle deflections are larger than that for longitudinal displacement correspondingly under the same conditions.

\subsection{Ultimate strain comparison for three typical faces}

Fig. 10 shows the observed ultimate strain against the slenderness ratio $(\lambda)$ for face A. The slenderness ratio $(\lambda)$ can be calculated by the following equations,

$$
\begin{gathered}
\lambda=l_{0} / i \\
i=\sqrt{I / A}
\end{gathered}
$$

Where, $l_{0}$ is the calculation length of the LBL column; $i$ is the gyration radius of the cross section; $I$ is the inertia moment of the cross section and $A$ is the area of the cross section.

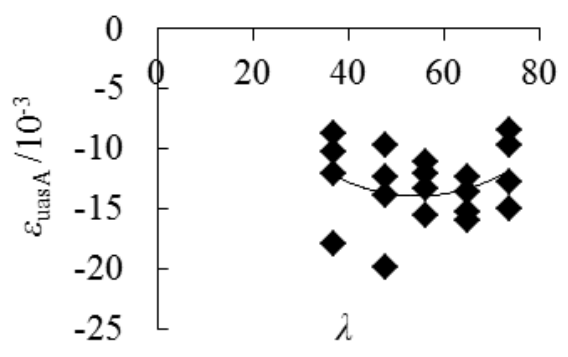

(a) Longitudinal strain

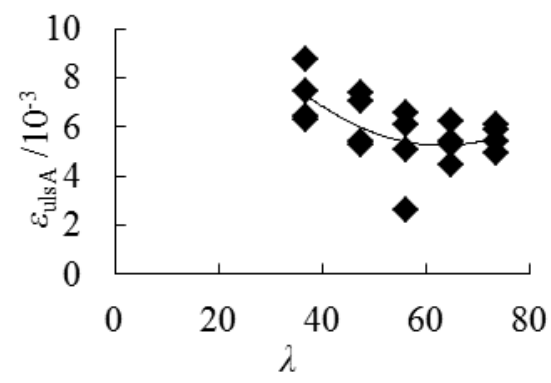

(b) Lateral strain

Fig. 10. Ultimate strain vs slenderness ratio $(\lambda)$ for face A

The absolute longitudinal strain values increase firstly until slenderness ratio reaches a value of 60 or so and then decrease. While the absolute lateral strain values decrease until slenderness ratio reaches a value of 60 or so and then increase but not very much. Regression analysis resulted in the following relationships which could be used under the given circumstances to predict strains from a slenderness ratio.

$$
\begin{array}{cc}
\varepsilon_{\text {uasA }}=0.0056 \lambda^{2}-0.61 \lambda+2.62 & \left(\mathrm{R}^{2}=0.078\right) \\
\varepsilon_{\text {ulsA }}=0.003 \lambda^{2}-0.376 \lambda+17.14 & \left(\mathrm{R}^{2}=0.362\right)
\end{array}
$$

Where, $\varepsilon_{\text {uasA }}$ is the ultimate axial strain for face A of the LBL column $\left(10^{-3}\right) ; \varepsilon_{\text {ulsA }}$ is the ultimate lateral strain for face A of the LBL column $\left(10^{-3}\right)$. 


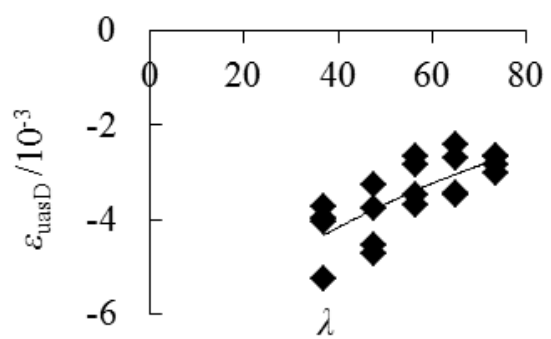

(a) Longitudinal strain

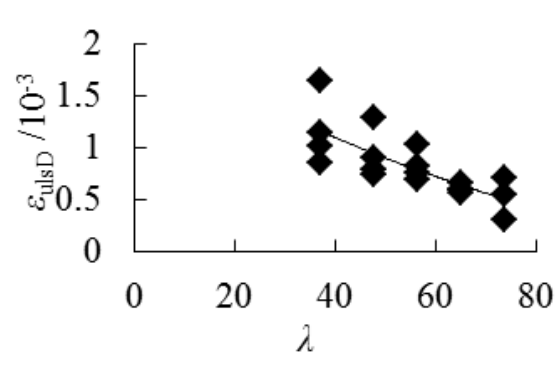

(b) Lateral strain

Fig. 11. Ultimate strain vs slenderness ratio $(\lambda)$ for face $D$

Fig. 11 shows the ultimate strain against the slenderness ratio for face D. Both the absolute longitudinal strain values and lateral strain values decrease with the increase of slenderness ratio for the test specimens. And all these values are smaller than the corresponding values for face A. Regression analysis resulted in the following relationships which could be used under the given circumstances to predict strains from a known eccentricity.

$$
\begin{array}{ll}
\varepsilon_{\text {uasD }}=-0.0003 \lambda^{2}+0.077 \lambda-6.78 & \left(\mathrm{R}^{2}=0.556\right) \\
\varepsilon_{\text {ulsD }}=0.00008 \lambda^{2}+0.027 \lambda-2.03 & \left(\mathrm{R}^{2}=0.576\right)
\end{array}
$$

Where, $\varepsilon_{\text {uasD }}$ is the ultimate axial strain for face D of the LBL column $\left(10^{-3}\right) ; \varepsilon_{\text {ulsD }}$ is the ultimate lateral strain for face D of the LBL column $\left(10^{-3}\right)$.

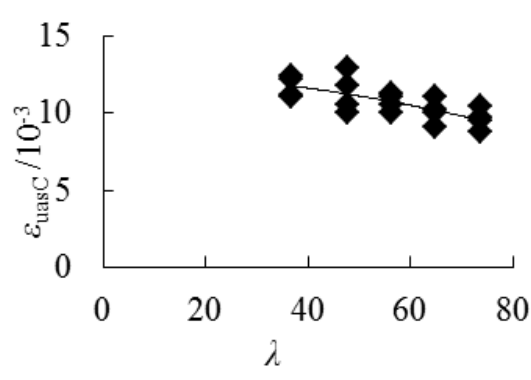

(a) Longitudinal strain

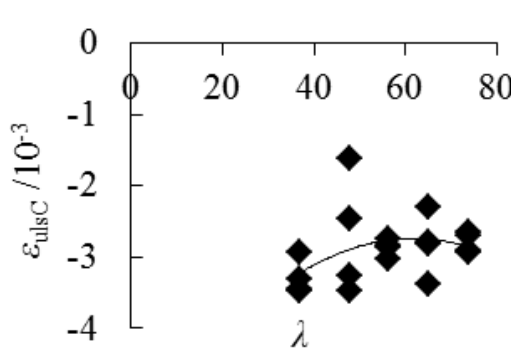

(b) Lateral strain

Fig. 12. Ultimate strain vs slenderness ratio $(\lambda)$ for face $C$

Figure 12 shows the variations in strains for face $C$. The absolute longitudinal strain values decrease slowly with an increase in the slenderness ratio. The absolute lateral strain values decrease firstly until the slenderness ratio reaches a value of 60 or so and then there is a small increase. The lateral strain values are more scattered than longitudinal strain values. Regression analysis was carried on the obtained test results, and following relationships can be used to predict strains on face $\mathrm{C}$ for the considered LBL columns.

$$
\begin{array}{cc}
\varepsilon_{\text {uas }}=-0.0005 \lambda^{2}-0.0093 \lambda+12.71 & \left(\mathrm{R}^{2}=0.529\right) \\
\varepsilon_{\text {ulsC }}=-0.0008 \lambda^{2}+0.0966 \lambda-5.72 & \left(\mathrm{R}^{2}=0.156\right)
\end{array}
$$


Where, $\varepsilon_{\text {uasC }}$ is the ultimate axial strain for face $\mathrm{C}$ of the LBL column $\left(10^{-3}\right) ; \varepsilon_{\text {ulsC }}$ is the ultimate lateral strain for face $\mathrm{C}$ of the LBL column $\left(10^{-3}\right)$.

\section{Combined analysis}

\subsection{Moment-curvature curves}

Based on equation (1), the curvature $(\phi)$ for the middle cross-section can be expressed as following

$$
\phi=1 / \rho=\pi^{2} w / L^{2}
$$

Where, $\rho$ is the curvature radius; $w$ is the middle lateral deflection.

Fig. 13 shows how the slenderness ratio influences the moment-curvature. It can be seen clearly that all specimens experienced three stages which were elastic, elastic-plastic, and cracking stage no matter how long the column was. There is excellent consistency in the results, particularly in during elastic response. After achieving the peak loads, all specimens cracked suddenly and the load values decreased in a short time.

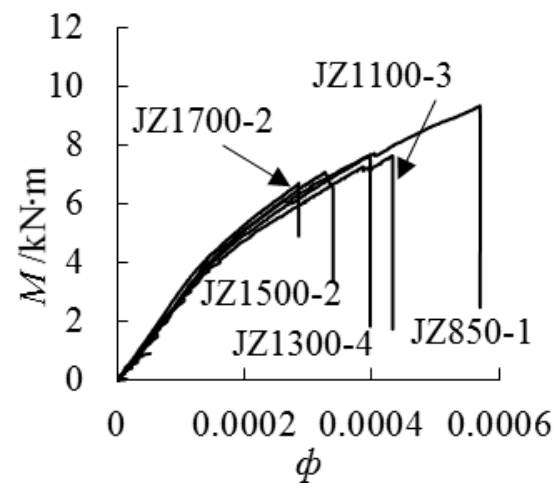

Fig. 13. Moment - curvature curves

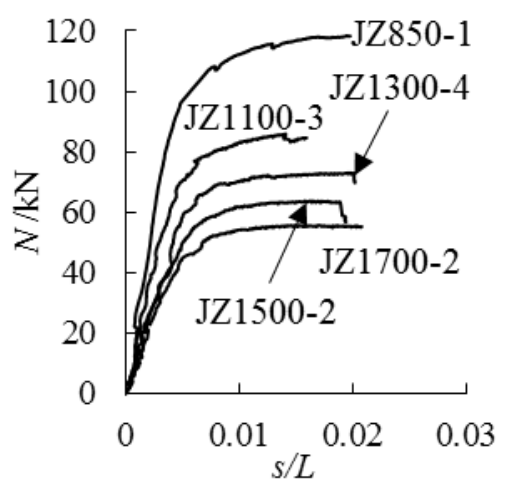

Fig. 14. Load - relative compression ratio curves

Fig. 14 shows typical load - relative compression ratio $(s / L)$ curves for all designed length for the columns. It can be seen clearly that both the ultimate load and the relative compression ratio decrease with the increasing of the slenderness ratios. Slenderness ratio is one of the main influencing factors for the mechanical properties of columns.

\subsection{Ultimate displacement comparison}

Fig. 15 shows the variations observed in lateral deflection at mid-height as well as in axial deformation at peak load for the slenderness ratios considered. Both the mid-height deflections and the axial deformation at peak load increases with increasing slenderness ratios. The ultimate axial deformation is more scattered than the mid-height deflections. By statistical regression from the test results, the following relationships under the condition mentioned previously can be expressed as

$$
\begin{gathered}
w_{\mathrm{ul}}=0.398 \lambda^{1.27} \quad\left(\mathrm{R}^{2}=0.937\right) \\
s_{\mathrm{ul}}=0.0021 \lambda^{2}+0.0407 \lambda+13.80 \quad\left(\mathrm{R}^{2}=0.711\right)
\end{gathered}
$$


Where, $w_{\mathrm{ul}}$ is the ultimate middle deflection of the LBL column $(\mathrm{mm}) ; s_{\mathrm{ul}}$ is the ultimate axial deformation of the LBL column (mm).

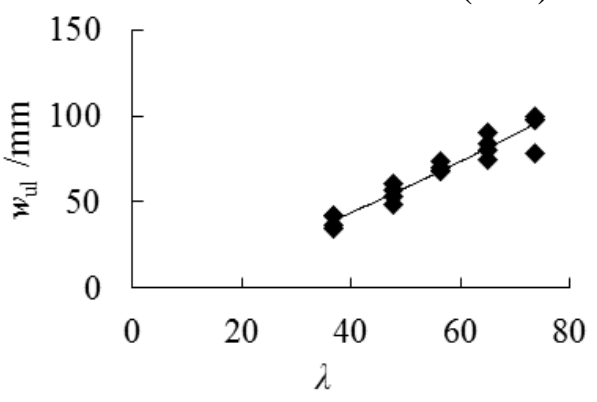

(a) Ultimate mid-height deflection

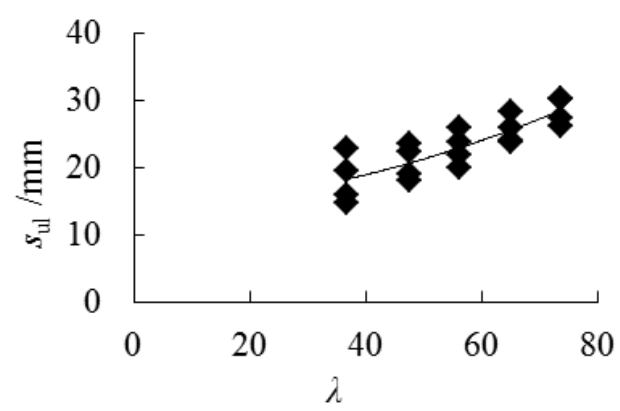

(b) Ultimate axial deformation

Fig. 15. Relationship between the displacement and slenderness ratio

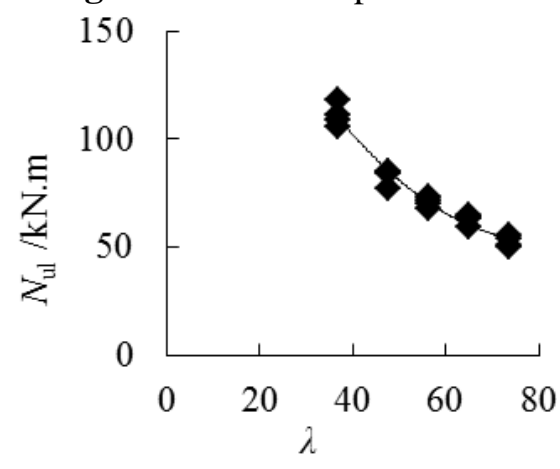

(a) Ultimate load

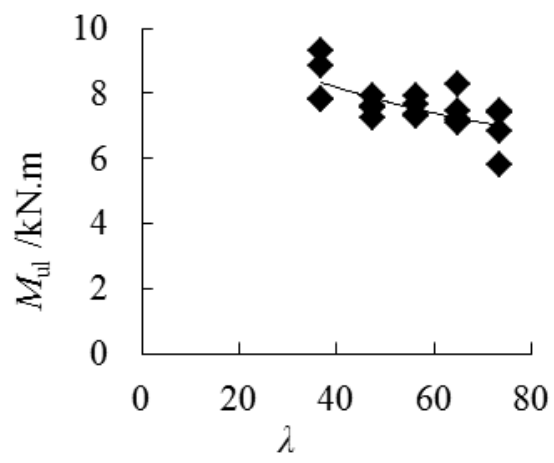

(b) Ultimate moment

Fig. 16. Ultimate bearing capacity comparison

Fig. 16 shows the ultimate load-bearing capacity (including load and moment) comparison among different group specimens with different slenderness ratios. The ultimate load values decrease quickly with the increase of the slenderness ratios in the beginning, and then decrease slowly. However, the ultimate moment decreases slowly as the slenderness ratios become larger. The slenderness ratio is the main influencing factor on the load-bearing capacity of the columns. By statistical regression from the test results, the relationship between the bearing capacity and slenderness ratios under the condition mentioned previously can be expressed as

$$
\begin{array}{cc}
N_{\mathrm{ul}}=0.029 \lambda^{2}-4.72 \lambda+244.37 & \left(\mathrm{R}^{2}=0.97\right) \\
M_{\mathrm{ul}}=0.0003 \lambda^{2}-0.0729 \lambda+10.57 & \left(\mathrm{R}^{2}=0.441\right)
\end{array}
$$

Where, $N_{\mathrm{ul}}$ is the ultimate load of the LBL column $(\mathrm{kN}) ; M_{\mathrm{ul}}$ is the ultimate moment of the LBL column (kN.m).

\subsection{Influencing coefficient of slenderness ratio}

As discussed before, slenderness ratio is one of the main influencing factors on the mechanical properties of LBL column. In order to consider this effect, the slenderness ratio 
influencing coefficient $\varphi_{\lambda}$ was proposed for calculating the ultimate bearing capacity and could be expressed as following,

$$
\varphi_{\lambda}=N_{\mathrm{ul}} / N_{0}
$$

Where, $N_{\text {ul }}$ is the ultimate bearing capacity of LBL columns under eccentric compression;

$N_{0}$ the ultimate bearing capacity of LBL columns for group JZ850.

In reality, there is interaction between the slenderness ratio influencing coefficient and geometric parameters of the laminated bamboo lumber columns. Combining with the numerical analysis and then reevaluating the constant coefficients by statistical regression on the whole test data set, it is found that the values for $1 / \varphi_{\lambda}$ increase approximately linear with the increasing of the slenderness ratio. Based on this discover, an equation for calculating the slenderness ratio influencing coefficient $\varphi_{\lambda}$ of LBL columns can be expressed as

$$
\varphi_{\lambda}=\frac{1}{0.029 \lambda-0.0638}
$$

Where, $\lambda$ is the slenderness value of the LBL column.

According to formula (15), the ultimate bearing capacity can be calculated by the following equation

$$
N_{\mathrm{ul}}=\varphi_{\lambda} N_{0}
$$

Where, the slenderness ratio coefficient $\varphi_{\lambda}$ can be calculated by equation (16).

The test results and calculation results by equation (17) for each group of specimens, in the form of mean, standard deviation (SD) and Coefficients of variation (COV) values are summarized in Table 2. $N_{\mathrm{ul}}^{\mathrm{t}}$ is the average test ultimate load and $N_{\mathrm{ul}}^{\mathrm{c}}$ is the calculation ultimate load from equation (17). $\mu$ stands for the mean value of $N_{\mathrm{ul}}^{\mathrm{t}} / N_{\mathrm{ul}}^{\mathrm{c}}$. It can be seen clearly that both the standard deviation coefficients and the coefficients of variation are less than 0.050 . That is to say, the calculation results obtained from the equation give a good agreement with the test results.

Table 2

Comparison between the test results and calculation results

\begin{tabular}{cccccccc}
\hline Group & $\lambda$ & $\varphi_{\lambda}$ & $N_{\mathrm{ul}}^{\mathrm{t}}(\mathrm{kN})$ & $N_{\mathrm{ul}}^{\mathrm{c}}(\mathrm{kN})$ & $\mu$ & $\mathrm{SD}$ & $\mathrm{COV}$ \\
\hline JZC850 & 36.8 & 0.984 & 111.1 & 110.7 & 1.004 & 0.048 & 0.0479
\end{tabular}




\begin{tabular}{llllllll} 
JZC1100 & 47.6 & 0.760 & 82.8 & 84.3 & 0.982 & 0.0457 & 0.0465 \\
JZC1300 & 56.3 & 0.643 & 70.7 & 70.8 & 0.999 & 0.0311 & 0.0311 \\
JZC1500 & 65.0 & 0.558 & 63.0 & 61.0 & 1.033 & 0.0376 & 0.0364 \\
JZC1700 & 73.6 & 0.492 & 52.7 & 53.6 & 0.982 & 0.0458 & 0.0466 \\
\hline
\end{tabular}

All the equations presented in this paper show how the factors investigated were found to influence the mechanical performance of columns under eccentric compression with different slenderness ratio in this particular series of the tests, and can be used as reference for further work to establish a generally applicable formula for code adoption. Previous research by $\mathrm{Li}$ et al (2016a, 2016b, and 2016c) have focused on the effect of the two tangential directions and different eccentricity values on the behaviour of LBL columns. However, these are just a few of the many factors which can influence the mechanical properties of LBL columns, such as bamboo species, production batches, and sample size among other. For this reason, more experimental studies and theoretical analyses are needed to derive suitable expressions for codes of practice.

\section{Conclusions}

In order to investigate how the slenderness ratio influences the mechanical properties of LBL columns under eccentric compression, 20 column tests have been carried out. Based on the analysis of the test data, the following conclusions can be drawn.

(1) Two main failure models can be classified based on the test results for the LBL columns. The main failure phenomenon for failure mode $I$ is that the specimen splits firstly on the tensile side, while for failure mode II no obvious cracks appear on the surface of the specimens until the end of the test.

(2) Irrespective of the column length, in most cases the ultimate values of both longitudinal and lateral strain for compression face A are larger than the corresponding values for tensile face $\mathrm{C}$.

(3) All specimens experienced three stages which were elastic, elastic-plastic, and cracking stage no matter how long the column was.

(4) Irrespective of the column length, the strain across the cross-section of the LBL column for each specimen is basically linear throughout the loading process, following standard normal section bending theory.

(5) Based on the test results, a relationship between the ultimate mechanical strength and slenderness ratio was proposed. All the equations presented in this paper can be used as reference for further work to establish a generally applicable formula for code adoption.

(6) Combining all the test data, an equation for calculating a slenderness ratio influencing coefficient $\varphi_{\lambda}$ of LBL columns is proposed. The calculation results obtained from the proposed equations give a good agreement with the test results.

\section{Acknowledgments}


The material presented in this paper is based upon work supported by the Open Fund Project from Key Laboratory of Concrete and Pre-stressed Concrete Structure of Ministry of Education (Southeast university), the China Postdoctoral Science Foundation (2015M580382), Jiangsu Postdoctoral Science Foundation Project (1501037A), the National Natural Science Foundation of China (51308301), the Natural Science Foundation of Jiang-su Province (No. BK20130978), and a Project Funded by the Priority Academic Program Development of Jiangsu Higher Education Institutions. Any opinions, findings, and conclusions or recommendations expressed in this material are those of the writer(s) and do not necessarily reflect the views of the foundations. The writers gratefully acknowledge Qi-jun WANG, Zhuang-yan SHEN, Hao YANG, Ming-lei YAO, Shuai-hong ZHANG, Jin-yuan WANG, Zhi-hao YIN, Jin-xiu SUN, Wei-xu ZHU and others from the Nanjing Forestry University for helping with the tests.

\section{References}

Correal JF, and Ramirez F (2010) Adhesive bond performance in glue line shear and bending for glued laminated guadua bamboo. Journal of Tropical Forest Science 22(4): 433-439.

Correal JF., Echeverry JS., Ramírez F, Yamín LE (2014) Experimental evaluation of physical and mechanical properties of Glued Laminated Guadua angustifolia Kunth. Construction and Building Materials 73: 105-112.

Chen G, Zhou T, Li C, Zhang Q, Li H (2016) Experimental study on the OSB webbed bamboo beams. Journal of Nanjing Forestry University (Natural Science Edition) 40(05): 121-125.

Huang M, Zhang X, Yu W, Li W, Liu X (2016) Mechanical properties and structure characterization of bamboo softened by high temperature steam. Journal of Forestry Engineering 1(4):64-68.

Lee AWC, Bai X, and Bangi AP (1998) Selected properties of laboratory-made laminated-bamboo lumber. Holzforschung 52(2): 207-210.

Lee CH, Chung M, Lin C, and Yang T (2012) Effects of layered structure on the physical and mechanical properties of laminated moso bamboo (Phyllostachys edulis) flooring. Construction and Building Materials 28(1): 31-35.

Li Y, Xu B, Zhang Q, Jiang S (2016a) Present situation and the countermeasure analysis of bamboo timber processing industry in China. Journal of Forestry Engineering 1(1):2-7.

Li H, Zhang Q, Xiong X (2016b) Review on laminated bamboo lumber. Journal of Forestry Engineering 1(6): 110-116.

Li H, Deeks AJ, Zhang Q, Wu G (2016c) Flexural performance of laminated bamboo lumber Beam. BioResources 11(1): 929-943.

Li H, Chen G, Zhang Q, Ashraf M, Xu B, \& Li Y (2016d) Mechanical properties of laminated bamboo lumber column under radial eccentric compression. Construction and Building Materials 121: 644-652.

Li H, Wu G, Zhang Q, \& Su J (2016e) Mechanical evaluation for laminated bamboo lumber along two eccentric compression directions. Journal of wood science 62(6): 503-517.

Li H, Wu G, Zhang Q, \& Chen G (2016f) Experimental study on side pressure LBL under tangential eccentric compression. Journal of Hunan University (Natural Science) 43(5): 90-96. 
Li H, Su J, Deeks AJ, Zhang Q, Wei D, \& Yuan C (2015a) Eccentric compression performance of parallel bamboo strand lumber column. BioResources 10(4): 7065-7080.

Li H, Su J, Zhang Q, Deeks AJ, \& Hui D (2015b) Mechanical performance of laminated bamboo column under axial compression. Composites Part B: Engineering 79: 374-382.

Luna P, Takeuchi C, Alvarado C, \& Moreno I (2013) Glued Laminated Guadua angustifolia Bamboo Columns// Acta Horticulturae. Belgium: Int Soc Horticultural Science, 125-129.

Li H, Wu G, Zhang Q, Deeks AJ, Su J (2018) Ultimate bending capacity evaluation of laminated bamboo lumber beams. Construction and Building Materials 121: 644-652.

Li H, Zhang Q, Huang D, \& Deeks AJ (2013) Compressive performance of laminated bamboo. Composites Part B: Engineering 54: 319-328.

Mahdavi M, Clouston PL, and Arwade SR (2011) Development of laminated bamboo lumber: Review of processing, performance, and economical considerations. Journal of Materials in Civil Engineering 23(7): 1036-1042.

Mahdavi M, Clouston PL, Arwade SR (2012) A low-technology approach toward fabrication of Laminated Bamboo Lumber. Construction and Building Materials 29: 257-262.

Nugroho N, and Ando N (2001) Development of structural composite products made from bamboo. II. Fundamental properties of laminated bamboo lumber. Journal of Wood Science 47(3): 237-242.

Sharma B, Gatóo A, Ramage M (2015) Effect of processing methods on the mechanical properties of engineered bamboo. Construction and Building Materials 83: 95-101.

Sharma B, Bauer H, Schickhofer G, Ramage M (2017) Mechanical characterisation of structural laminated bamboo. Proceedings of the Institution of Civil Engineers-Structures and Buildings 170(4): 250-264.

Sulaiman O, Hashim R, and Wahab R (2006) Evaluation of shear strength of oil treated laminated bamboo. Bioresource Technology 97(18): 2466-2469.

Su J, Li H, Yang P, Zhang Q, \& Chen G. (2015a) Mechanical Performance Study on laminated bamboo lumber column pier under axial compression. China Forestry Science and Technology 29(5): 89-93.

Sulastiningsih IM, and Nurwati (2009) Physical and mechanical properties of laminated bamboo board. Journal of Tropical Forest Science 21(3): 246-251.

Su J, Wu F, Li H, \& Yang P (2015b) Experimental research on parallel bamboo strand lumber column under axial compression. China Sciencepaper 10(1): 39-41.

Verma CS, and Chariar VM (2012) Development of layered laminate bamboo composite and their mechanical properties. Composites Part B-Engineering 43(3): 1063-1069.

Wei Y, Zhou M, \& Yuan L (2016) Mechanical performance of glulam bamboo columns under eccentric loading. Acta Materiae Compositae Sinica 33(2): 379-385.

Xiao Y, Shan B (2013) Modern bamboo structures. China Architecture \& Building Press, Beijing, China.

Yeh MC, and Lin YL (2012) Finger joint performance of structural laminated bamboo member. Journal of Wood Science 58(2): 120-127.

Zhong Y, Ren H, Jiang Z. (2016) Effects of Temperature on the Compressive Strength Parallel to the Grain of Bamboo Scrimber. Materials 9(436): 1-9. 